\title{
Depletion of new neurons by image guided irradiation
}

\author{
Y.-F.Tan' ${ }^{1}$, S. Rosenzweig' ${ }^{1}$, D. Jaffray ${ }^{2}$ and J. M. Wojtowicz ${ }^{\text {* }}$ \\ Department of Physiology, Faculty of Medicine, University of Toronto, Toronto, ON, Canada \\ 2 Radiation Oncology, Princess Margaret Hospital, University of Toronto, Toronto, ON, Canada
}

\section{Edited by:}

Silvia De Marchis, University of Turin,

Italy

\section{Reviewed by:}

Tatsunori Seki, Tokyo Medical

University, Japan

Gilles Gheusi, Institut Pasteur, France

${ }^{*}$ Correspondence:

J. M. Wojtowicz, Department of

Physiology, University of Toronto,

Medical Sciences Building, Third Floor

1 King's College Circle, Toronto, ON,

Canada M5S 1 A8

e-mail:martin.wojtowicz@utoronto.ca
Ionizing radiation continues to be a relevant tool in both imaging and the treatment of cancer. Experimental uses of focal irradiation have recently been expanded to studies of new neurons in the adult brain. Such studies have shown cognitive deficits following radiation treatment and raised caution as to possible unintentional effects that may occur in humans. Conflicting outcomes of the effects of irradiation on adult neurogenesis suggest that the effects are either transient or permanent. In this study, we used an irradiation apparatus employed in the treatment of human tumors to assess radiation effects on rat neurogenesis. For subjects we used adult male rats (Sprague-Dawley) under anesthesia. The irradiation beam was directed at the hippocampus, a center for learning and memory, and the site of neurogenic activity in adult brain. The irradiation was applied at a dose-rate $0.6 \mathrm{~Gy} / \mathrm{min}$ for total single-fraction, doses ranging from 0.5 to $10.0 \mathrm{~Gy}$. The animals were returned to home cages and recovered with no sign of any side effects. The neurogenesis was measured either 1 week or 6 weeks after the irradiation. At 1 week, the number of neuronal progenitors was reduced in a dose-dependent manner with the $50 \%$ reduction at $0.78 \mathrm{~Gy}$. The dose-response curve was well fitted by a double exponential suggesting two processes. Examination of the tissue with quantitative immunohistochemistry revealed a dominant low-dose effect on neuronal progenitors resulting in $80 \%$ suppression of neurogenesis. This effect was partially reversible, possibly due to compensatory proliferation of the remaining precursors. At higher doses ( $>5 \mathrm{~Gy}$ ) there was additional, nearly complete block of neurogenesis without compensatory proliferation. We conclude that notwithstanding the usefulness of irradiation for experimental purposes, the exposure of human subjects to doses often used in radiotherapy treatment could be damaging and cause cognitive impairments.

\section{INTRODUCTION}

Ionizing radiation has emerged as a standard method in animal studies on functional effects of adult neurogenesis (Wojtowicz, 2006). It is the only method that is currently available for relatively uninvasive deletion of new neurons in selected brain regions in a dose-dependent manner. It can be used on any species and its effects are readily translated to humans (Monje et al., 2002; Monje and Palmer, 2003). With the advent of improved focusing methods combined with brain imaging the irradiation beam can be applied to brain targets as small as a few millimeters in diameter. In this study, we employed such an approach to construct a dose-response curve showing effects of irradiation on the population of neuronal progenitors in the hippocampus, a brain structure involved in spatial and episodic memory (Cohen and Eichenbaum, 1993). Previous studies generally used one or two doses and focused on the mechanisms of cell depletion. Several studies have established that within hours of the treatment many of the proliferating cells die through apoptosis (Peissner et al., 1999; Mizumatsu et al., 2003). This is consistent with the established vulnerability of the mitotically active cells to radiation. However, there are indications of other damaging processes as well. Inflammation with the resulting release of toxic cytokines (Monje et al., 2003), destruction of blood capillaries (Monje et al., 2003) and killing of quiescent stem cells (Encinas et al., 2008) have been reported. There are conflicting reports on irreversible and reversible nature of these effects (Tada et al., 2000;
Ben Abdallah et al., 2007). This is an important issue since behavioral tests require a stable baseline of neurogenesis at a given level, presumably lower than in the intact brain. If the effects of irradiation are in fact reversible the factors that can speed up or enhance its recovery are of importance in clinical practice where irradiation is used to treat malignant tumors often with unavoidable collateral damage on the surrounding tissue. If the effects are irreversible, the cause of the permanent damage should be understood since it could influence the mechanism of the remaining neurogenesis and the properties of the surviving neurons. This study shows that dose-dependence is bimodal and can be accounted for by low-dose reversible and high-dose irreversible effects of irradiation.

\section{METHODS \\ SUBJECTS}

Thirty 12-week-old male S-D rats (Charles River) were used in this study.

The animals were allowed to acclimate in the facility for 1 week after the delivery. All procedures were approved by the University of Toronto and University Hospital Network ethics committees.

\section{IRRADIATION}

Just prior to irradiation the animals were anesthetized with IP injection of ketamine/xylazine at $85 / 5 \mathrm{mg} / \mathrm{kg}$. Animals were placed one by one on the treatment couch of the irradiation unit (Elekta Synergy) 
and aligned at the intersection of isocenter marking lasers just in front of the ears (Figure 1). The shielded door to the treatment room was shut and the animal imaged using the integrated conebeam CT system (imaging dose of $<0.03 \mathrm{~Gy}$ ) to obtain an outline of the skull together with the body landmarks such as oral cavity, ear canals, etc. The graphical representation of the isocenter of the treatment beam was aligned with the approximate location of the hippocampus as determined from a pre-existing MRI and CT scan from a generic rat. The position of the rat with respect to the actual isocenter was then automatically adjusted using the robotic table of the treatment unit. The dosimetry with the resulting fields as seen from the side and from the front was done using Pinnacle software (Figure 2). Thus the 98, 80, 60\%, isodose level were seen superimposed on the image of the skull aligned with the approximate location of the hippocampus. The dose-rate of irradiation was $0.6 \mathrm{~Gy} / \mathrm{min}$ and the total dose was obtained by irradiating equally from each side (left and right lateral beams). Doses were applied

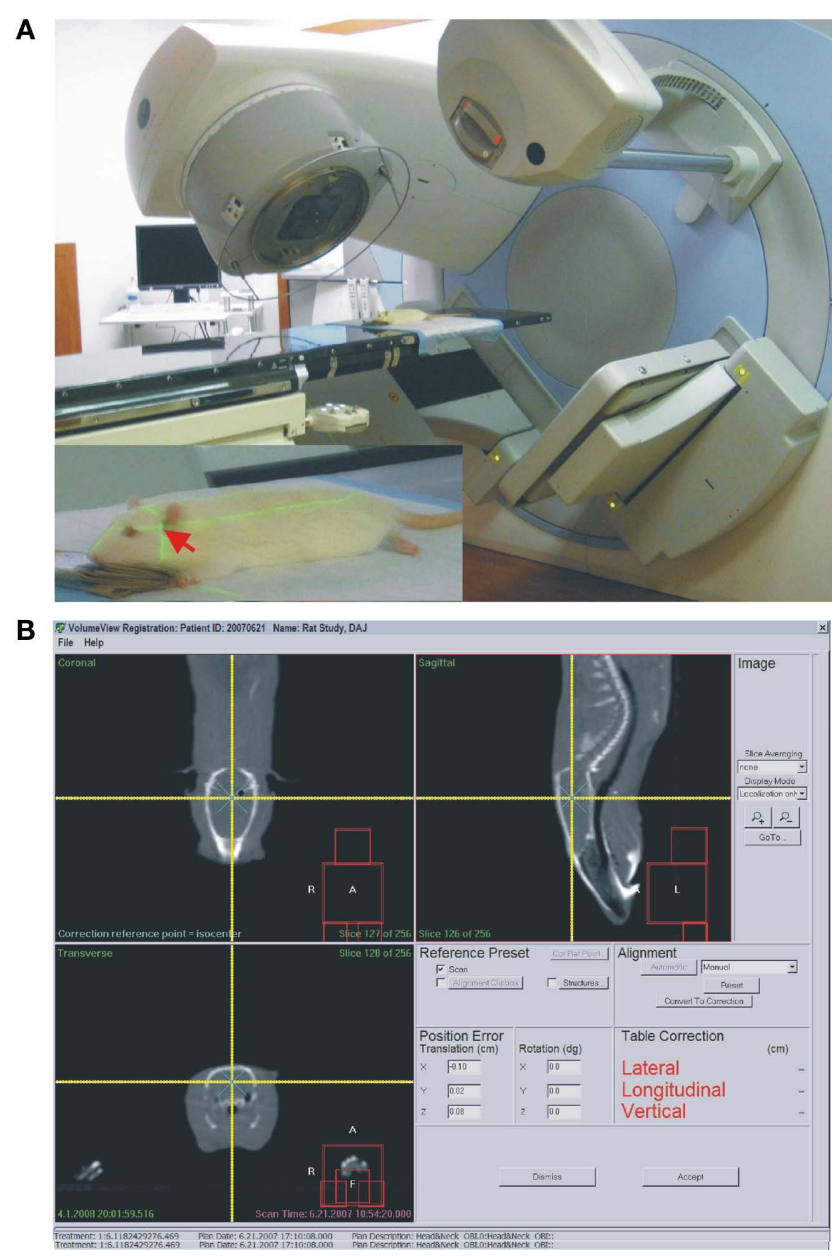

FIGURE 1 | (A) Elekta synergy CT system used for imaging and irradiation in this study. Same system is also used for human radiotherapy. Inset. Red arrow points at the approximate location of the hippocampus at the intersection of the two guiding laser beams. (B) Screen-print of the targeting and dosimetry software allowing visualization of the rat's skull (white outline). The intersection of two yellow beams indicates the center point of the irradiation (isocenter). over the range from 0.5 to 10 Gy with three animals per dose. The treatment unit is referenced to the National Research Council of Canada (NRCC) radiation dosimetry standards through the

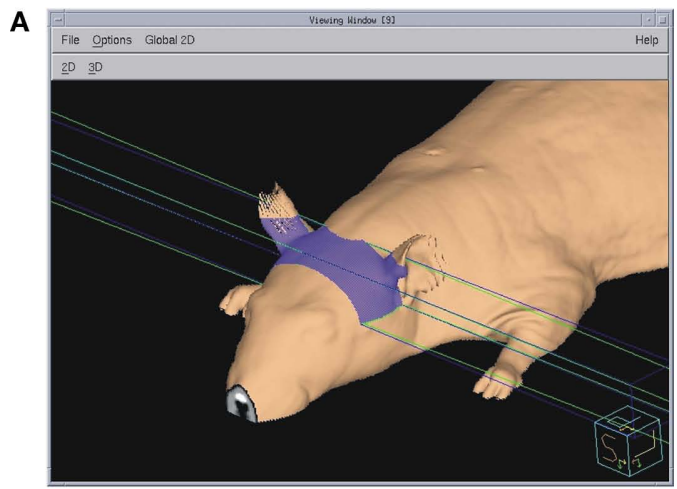

B

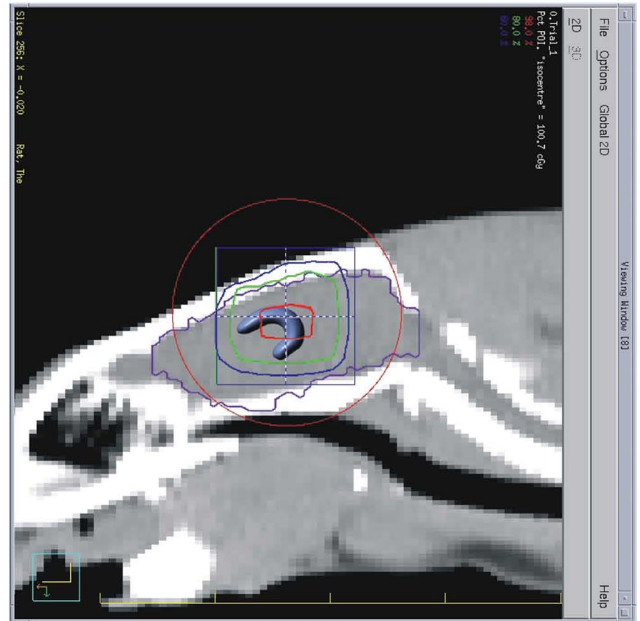

C

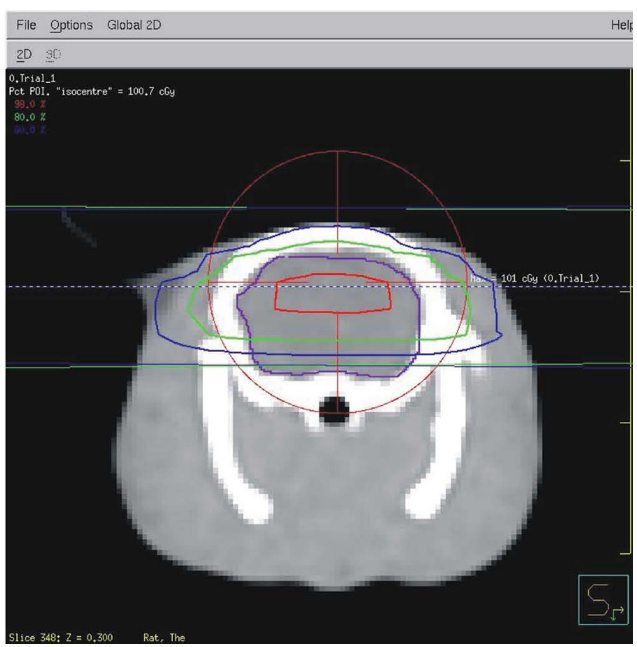

FIGURE 2 | (A) Dosimetry data show approximate irradiated area on the animal's skin. (B) Saggital CT scan with $98 \%$ dose (red), $80 \%$ dose (green), and $60 \%$ dose (blue) outlines. Brain outline is shown in purple and the hippocampus is sketched in the location determined from a brain atlas. Red circle and blue square are part of the targeting software with cross-hairs (visible only with zoom-in) at the isocenter. (C) Frontal view with the same isodose outlines. 
American Association of Physicists in Medicine TG-51 dosimetry protocol. Monitor units used for the irradiation were derived from the clinical dosimetry database of the Princess Margaret Hospital's Radiation Medicine Program. Sham controls were anesthetized but not imaged or irradiated. Following treatment, the animals were transferred to home cages and kept for 1 week or 6 weeks in standard conditions. There was no effect of the treatment on animal's behavior and no side effects such as hair loss or weight loss. The weights of the control animals $(n=3)$ were 394.7 before, and $425.7 \mathrm{~g}$ 1 week after treatment. The weights of the $10 \mathrm{~Gy}$ (the highest dose) irradiated animals $(n=3)$ were $392.7 \mathrm{~g}$ before treatment and $425.7 \mathrm{~g}$ 1 week after treatment.

\section{BrdU ADMINISTRATION}

5-Bromo-2-deoxyuridine (BrdU; Sigma, St. Louis, MO, USA) was dissolved in phosphate-buffered saline (PBS) containing $10 \mathrm{mM}$ $\mathrm{NaOH}$ for a final concentration of BrdU $20 \mathrm{mg} / \mathrm{ml}$. The solution was injected i.p. at a dose of $200 \mathrm{mg} / \mathrm{kg}$ of body weight at $9 \mathrm{AM}$ and again at $9 \mathrm{PM}$ on the 14th day after irradiation. The dose of BrdU was chosen to allow for maximal labeling of cells born during a 24-h period according the previously described procedures (McDonald and Wojtowicz, 2005).

\section{PERFUSION AND TISSUE PREPARATION}

Most animals were perfused at 1 week after irradiation with three animals per irradiation dose. This time point was chosen on the basis of published data showing a peak of proliferation following cell birth (McDonald and Wojtowicz, 2005). Several additional animals were perfused at 6 weeks, allowing measurement of cell survival and examination of long-term effects of irradiation on cell differentiation and maturation. Following intracardiac perfusion with PBS and 4\% paraformaldehyde, the right hippocampus was sectioned into three parts - dorsal, medial, and ventral - and post-fixed overnight in $4 \%$ paraformaldehyde at $4^{\circ} \mathrm{C}$. Serial $30 \mu \mathrm{m}$ sections of all segments were cut with a vibratome resulting in 200-250 sections per animal. One in 10 transverse sections were sampled using a systematic random sampling procedure along the dorso-ventral extent of the hippocampus.

\section{IMMUNOHISTOCHEMISTRY}

Procedures for detection of calbindin (CaBP), doublecortin (DCX), Ki67, and BrdU were the same as described in several research and methods articles recently published by us (Kee et al., 2002; McDonald and Wojtowicz, 2005; Wojtowicz and Kee, 2006). Primary antibodies, dilutions, and incubation times were as follows: CaBP (Chemicon AB1778), 1:200, $72 \mathrm{~h}$ at $4^{\circ} \mathrm{C}$; DCX (Santa Cruz; C-18, sc-8066), 1:200, 24 h at $4^{\circ} \mathrm{C}$; Ki67 (Vector labs. VP-K451), 1:200, $18 \mathrm{~h}$ at room temp.; BrdU (Cedarlane, OBT0030), 1:200, $24 \mathrm{~h}$ at $4^{\circ} \mathrm{C}$ ). The secondary antibodies were tagged with Alexa 594 (red) or 488 (green) fluorophores (Molecular Probes). Selected sections were stained for a microglia marker ED1 as described previously (Tan et al., 2010).

\section{DATA ANALYSIS}

Cells were counted in each of the sampled sections (20-25 sections per animal for each marker at each time point). In all experiments with 1-week survival, the cell counts were expressed per dentate gyrus (unilateral) by counting all labeled cells in the subgranular zone of the dentate gyrus in sampled sections and multiplying the average by the total number of sections per animal. In experiments with 6-weeks survival, the cells were counted per section, since we wished to compare three regions (dorsal, middle, and ventral) of the dentate gyrus with regard to effects of radiation. In these experiments, BrdU/CaBP double-labeled cells were examined under a confocal microscope (Leica, TC, SSL).

Statistical analysis was done using SigmaPlot 8 and SigmaStat 3.0 software. Data sets were analyzed with one-way or two-way ANOVA, as appropriate, and passed the normality and equal variance tests. All values given in the text and in figures show mean and standard errors.

\section{RESULTS}

Post-irradiation status of neurogenesis was assessed 1 week after treatment. This time point was chosen because previous BrdU tracing experiments revealed maximal density of the immature and proliferating cells at that time (McDonald and Wojtowicz, 2005). In addition, brain trauma, such as ischemia, produces maximal rebound neurogenesis 1 week after treatment (Tada et al., 2000; Dash et al., 2001; Sharp et al., 2002). Thus, the 1-week time point is an important milestone in normal and pathological cell development.

To examine possible effects of this procedure on neurogenesis we examined a wide range of doses, beginning with very low levels and spanning the previously used higher doses. As expected we observed strong inhibitory effect of high doses, but surprisingly even a low dose of 1 Gy had clear, pronounced effect (Figure 3). The irradiation produced statistically significant reductions in the total number of DCX+cells per dentate gyrus at all doses (ANOVA, with pairwise comparisons between the control and the irradiated groups, $P=0.001)$. We showed this decrease using another marker of neurogenesis, PSA-NCAM, that labels young neurons in the similar age range, but has very different chemical characteristics and entirely different cellular distribution (Seki and Arai, 1993). The controls and the 5 Gy-irradiated groups showed similar cell counts using DCX and PSA-NCAM labeling, 21.334 $\pm 1028,2.150 \pm 567$ for DCX and $21.308 \pm 654,1.839 \pm 652$ for PSA-NCAM, respectively. This confirmed that irradiation had a robust effect on cell number and was not marker-specific.

Densities of the proliferating Ki67+ cells in the same animals were not reduced at any of the doses. In fact, the proliferation appeared to be enhanced at the intermediate doses of 2, 3, and 4 Gy (ANOVA, $F=3.978, P=0.016$ ) compared to controls. These results suggest that reduced proliferation is either not the cause of lowered neurogenesis or that a delayed compensatory increase in proliferation occurred as a result of the initial insult. The observed increase in proliferation cannot instantly compensate for the underlying decrease in neurogenesis but could account for its recovery within a few weeks.

We next analyzed the dose-response relationship by fitting the DCX data to a double exponential curve with four parameters corresponding to the maxima and the rate constants of the two exponential components (Figure 4). A good fit to the data suggests two processes, one with a maximum of $80 \%$ suppression and sharp rising rate constant with a half dose of $0.78 \mathrm{~Gy}$, and the other with 


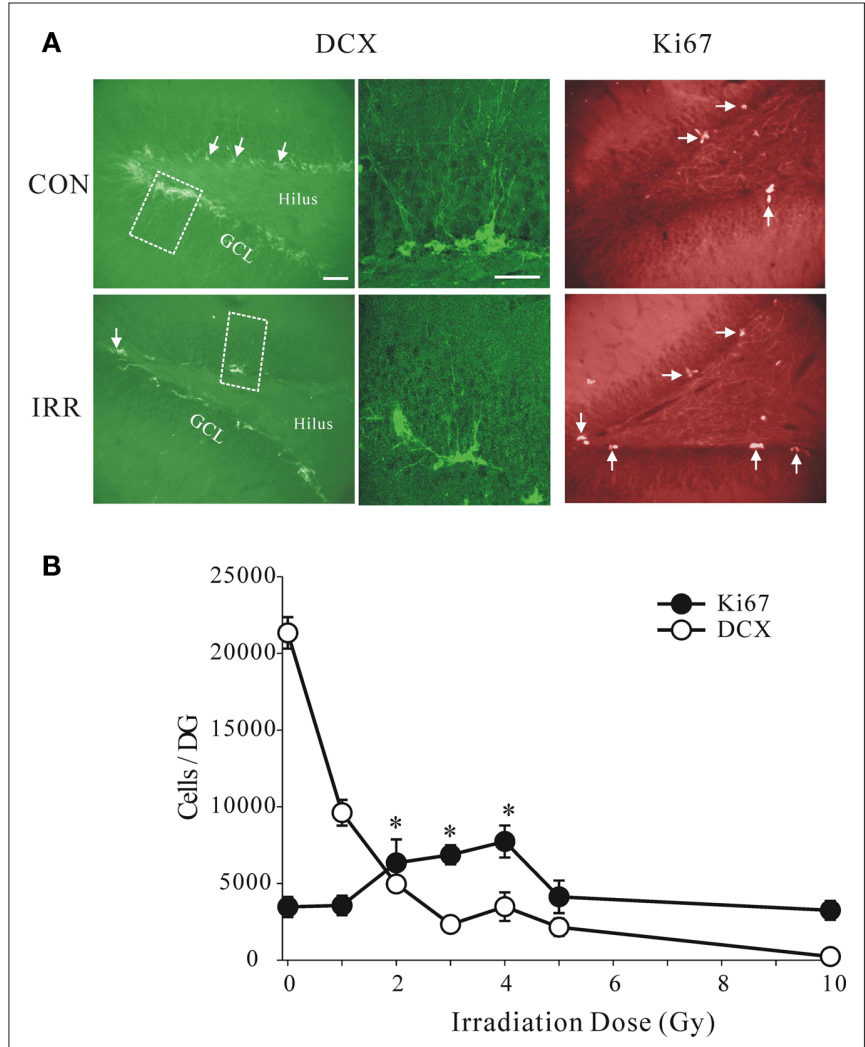

FIGURE 3 | (A) Images of transverse sections through the dentate gyrus stained for doublecortin (DCX), a marker of neuroblasts and young neurons, and for Ki67, a marker for proliferating cells. Note that the number of DCX-positive cells was reduced by irradiation (IRR), while the number of Ki67-positive cells was increased. The enlarged areas (broken line) are shown in the images in the middle showing typical morphology of normal young neurons in control animal and the immature morphology in irradiated animal. Dose of irradiation was $4 \mathrm{~Gy}$ and the survival time was 1 week after treatment. Calibration bars are $50 \mu \mathrm{m}$. (B) Absolute numbers of DCX+ and Ki67+cells per dentate gyrus at different doses. Note sharp dose-response dependence at low doses and a near complete depletion of DCX+cells at the highest dose (10 Gy). The number of proliferating cells was significantly increased at 2, 3, and $4 \mathrm{~Gy}$ (asterisks, ANOVA, $P=0.016$ ), but there was no change at the lowest and the highest doses.

the $100 \%$ suppression and half maximal dose of 45.5 Gy. Together, they completely account for the data and suggest a bimodal mechanism. Transient inflammation has been reported after irradiation (Monje et al., 2003; Noonan et al., 2010) at the doses similar to the ones used in this study. However, none of our animals tested at 1 week or 6 weeks post-irradiation showed any sign of inflammation even at the highest irradiation dose of 10 Gy (Figure 4B). The tissue from the study by Tan et al. (2010) where global ischemia induced prominent activation of microglia in CA1 and the Hilus was used as positive control. Thus, neither the reversible nor irreversible effects of irradiation were directly related to inflammation. Examination of the dorsal, medial and ventral thirds of the dentate gyrus showed no differences in the proportional effects of irradiation on Ki67+ or DCX+cells. Thus all data are reported as average changes per whole dentate gyrus. This is specifically illustrated for the 6-week data set (Figure 6, below), but also applicable to all data sets in this study.

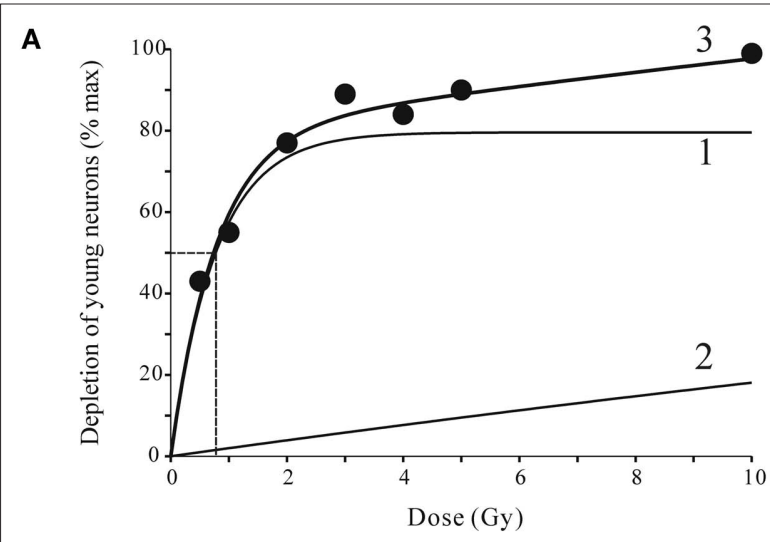

B Inflammation

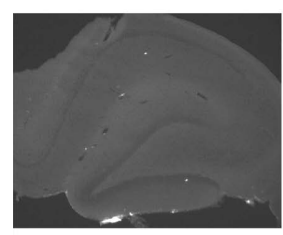

$\mathrm{CON}$

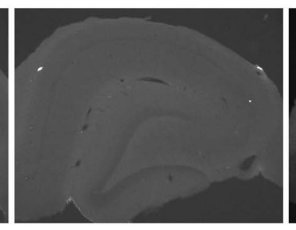

10 Gy

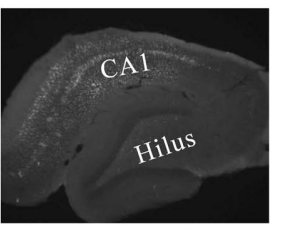

Ischemia
FIGURE 4 | (A) Quantitative dose-response relationship for effects of radiation on DCX-positive young neurons. The data points were fitted to a double exponential (3) with a fast (1) and a slow (2) component. Half-maximal dose of $0.78 \mathrm{~Gy}$ is shown by dashed lines. The parameters were: $\max _{1}=79.6$,

$a_{1}=1.28 . \max _{2}=99.7, a_{2}=0.022$. Regression analysis $F_{3,6}=32.06, P=0.0088$ (B) Representative images of tissue sections from control and $10 \mathrm{~Gy}$ irradiated animals at 1-week time point stained for microglia inflammation marker ED1. There was no detectable staining even at the highest dose (10 Gy) used in this study. For positive control the tissue from another study (Tan et al., 2010) shows clear microglia signal in CA1 and the Hilus 1 week after global ischemia.

We investigated the possibility that increased proliferation at moderate doses of irradiation can compensate for the loss of DCX+ cells. In this experiment we exposed the animals to $3 \mathrm{~Gy}$, which at 1 week causes suppression of neurogenesis by nearly $90 \%$ but increases proliferation by over two-fold. We hypothesized that with time the increased proliferation of the precursors should compensate for the initial cell loss. The results are consistent with this hypothesis. The number of Ki67+ cells was significantly higher in the irradiated animals at 1 week post-irradiation. Two-way ANOVA, taking time ( 1 or 6 weeks) and treatment (control or irradiation) as variables, shows a significant effect of time $\left(F_{1,11}=39.26\right.$, $P<0.001)$ and treatment $\left(F_{1,11}=18.06, P=0.003\right)$ and significant interactions $\left(F_{1,11}=8.86, P=0.018\right)$. In particular, with regard to the above hypothesis, the treatment had a significant effect at 1 week $(P<0.0001)$ but not at 6 weeks $(P=0.39)$. In contrast, the number of DCX+ cells was significantly reduced at 1-week time point. Two-way ANOVA shows a significant effect of time $\left(F_{1,11}=32.63, P<0.001\right)$ and treatment $\left(F_{1,11}=128.73, P<0.001\right)$ and significant interactions $\left(F_{1,11}=76.63, P<0.001\right)$. In particular, the treatment had a significant effect at 1 week $(P<0.0001)$ but not at 6 weeks $(P=0.10)$. These data are presented graphically using the relative values (irradiated/control) to show the recovery of the effects at 6 weeks. The absolute values for cell numbers are given in the legend of Figure 5. 

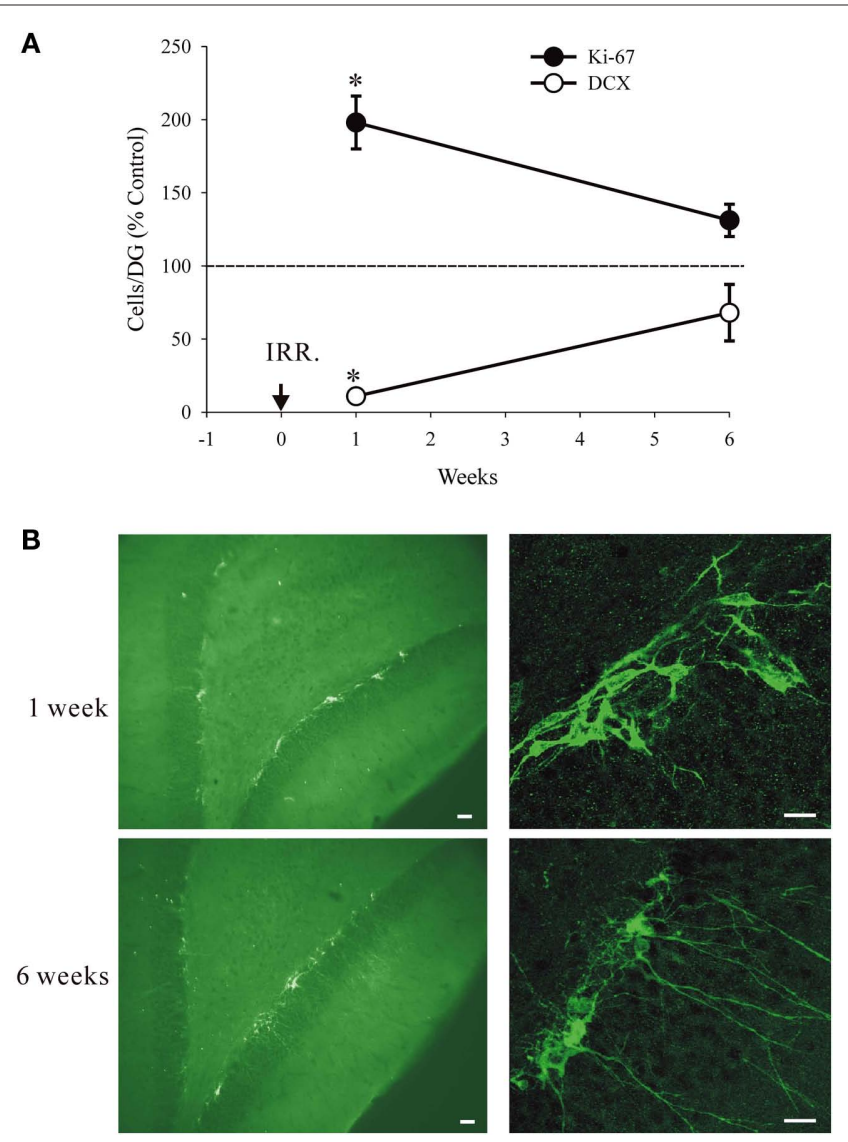

FIGURE 5 | Recovery from irradiation at 3Gy. (A) Relative numbers of DCX+ and Ki67+ cells at 1 week and at 6 weeks after treatment (IRR). The numbers of DCX+cells and Ki67+ cells at 1 week, but not at 6 weeks were significantly different from controls (asterisks). The absolute values for cell numbers per DG were as follows: For Ki67 at 1 week $3.464 \pm 657.6$ (controls) and $6.860 \pm 1.084 .3$ (irradiated). For Ki67 at 6 weeks $1.919 \pm 204.3$ (controls) and $2.516 \pm$ (irradiated). For DCX at 1 week $21.334 \pm 1.781 .4$ (controls) and $2.327 \pm 180.7$ (irradiated). For DCX at 6 weeks $7.654 \pm 979.7$ (controls) and $5.203 \pm 2562.1$ (irradiated). The reduced cell numbers at 6 vs. 1 week for controls are due to expected decline with animal's age. All values are means and standard errors ( $n=3$ in each group). (B) Typical immature appearance of the DCX+ cells at 1 week and a more normal appearance with longer dendrites at 6 weeks. Calibration bars are $20 \mu \mathrm{m}$ in all cases.

In the next experiment we showed that the remaining proliferation after irradiation resulted in the production of mature neurons, albeit at lower rates. Two groups of animals, 3 Gy irradiated and sham controls, were injected with BrdU at 2 weeks and killed at 6 weeks after irradiation, thus allowing 4 weeks for neuronal maturation of the BrdU-tagged neurons. The surviving neurons were co-labeled for BrdU and calbindin ( $\mathrm{CaBP})$, a neuronal marker that is expressed during neuronal maturation (McDonald and Wojtowicz, 2005). In this case we show separately the total number of BrdU+ and BrdU/CaBP+ cells and we include the distribution among the three hippocampal regions (Figure 6).

\section{DISCUSSION}

High energy radiation surrounds us in the environment, it is also used in high doses in ionizing X-ray procedures, various imaging devices such as CT scans, and in radiotherapy for treatment of cancer or benign conditions (Koh et al., 2006). One specialized application of the ionizing radiation is to inhibit growth of endogenous stem cells in adult brain in order to assess their function in normal physiology and in potential regeneration of the brain tissue (Saxe et al., 2006; Wojtowicz, 2006). The present study examined the effects of irradiation on the hippocampus, a key brain structure involved in learning and memory. There is now convincing evidence that inhibition of adult neurogenesis in the dentate gyrus, the integral part of the hippocampus, affects some types of learning and memory in experimental animals. In particular, the memory that relates past events to context is very sensitive to reduced neurogenesis (Winocur et al., 2006; Wojtowicz et al., 2008; Hernandez-Rabaza et al., 2009). With continuing use of the irradiation in animal experiments on neurogenesis it is important to understand the mechanisms and dose dependence of the treatment. It is also useful to assess the extent of cell depletion along the dorso-ventral axis of the hippocampus. Dorsal and ventral regions are unevenly involved in various behaviors and recent evidence suggests that neurogenesis is also polarized (Snyder et al., 2008). Our results show that the targeting procedure ensures uniform effects of radiation in all regions of the hippocampus along its dorso-ventral axis (Figure 6).

There exists conflicting evidence with regard to the duration of radiation effects. In most studies the effects were irreversible (Wojtowicz, 2006), but in others, either spontaneous or experimentally induced recovery has been observed (Monje et al., 2003; Rola et al., 2004; Ben Abdallah et al., 2007). Our new data reconcile these discrepancies by showing that the effects of irradiation are bimodal. There appear to be a low-dose effect of irradiation that is associated with enhanced compensatory proliferation of precursors and, a high dose effect that lacks such compensation. Most of previous reports of the irreversible effects on neurogenesis used the doses in the higher range, above $5 \mathrm{~Gy}$. The lower doses, below 5 Gy may be reversible by allowing sufficient time for recovery after treatment. However, it is also apparent that full recovery is unlikely since according to the exponential model shown in Figure 4A the high dose effect begins from 0 and grows steadily with dosage. This finding appears ominous for possible side effects of radiotherapy that uses large doses ( $>50$ Gy) in fractions of 1-2 Gy (Laack and Brown, 2004; Barani et al., 2007). It can also be expected that even some forms of imaging such as CT scans, that require significant exposure to radiation, may affect neurogenesis. It should be noted that the irreversible effects are not necessarily due to persistent inflammation since our irradiation procedure did not produce any detectable inflammation (Figure 4B). Unlike in our previous studies where we used pentobarbital anesthesia (Wojtowicz, 2006), the present study utilized a more common ketamine/xylazine anesthesia. Thus, the proposed protective action of the pentobarbital does not seem to be necessary to prevent the inflammation at least in the dose range utilized here.

The apparent increase in proliferation at lower doses (Figure 3) may seem paradoxical but in fact is in agreement with the well established compensatory or rebound response of proliferation to various types of brain injury including ischemia or mechanical trauma (Dash et al., 2001; Kee et al., 2001; Sharp et al., 2002). There is no doubt that proliferation is reduced within $24 \mathrm{~h}$ of the irradiation but the rebound at 1 week suggests a homeostatic mechanism that compensates for 

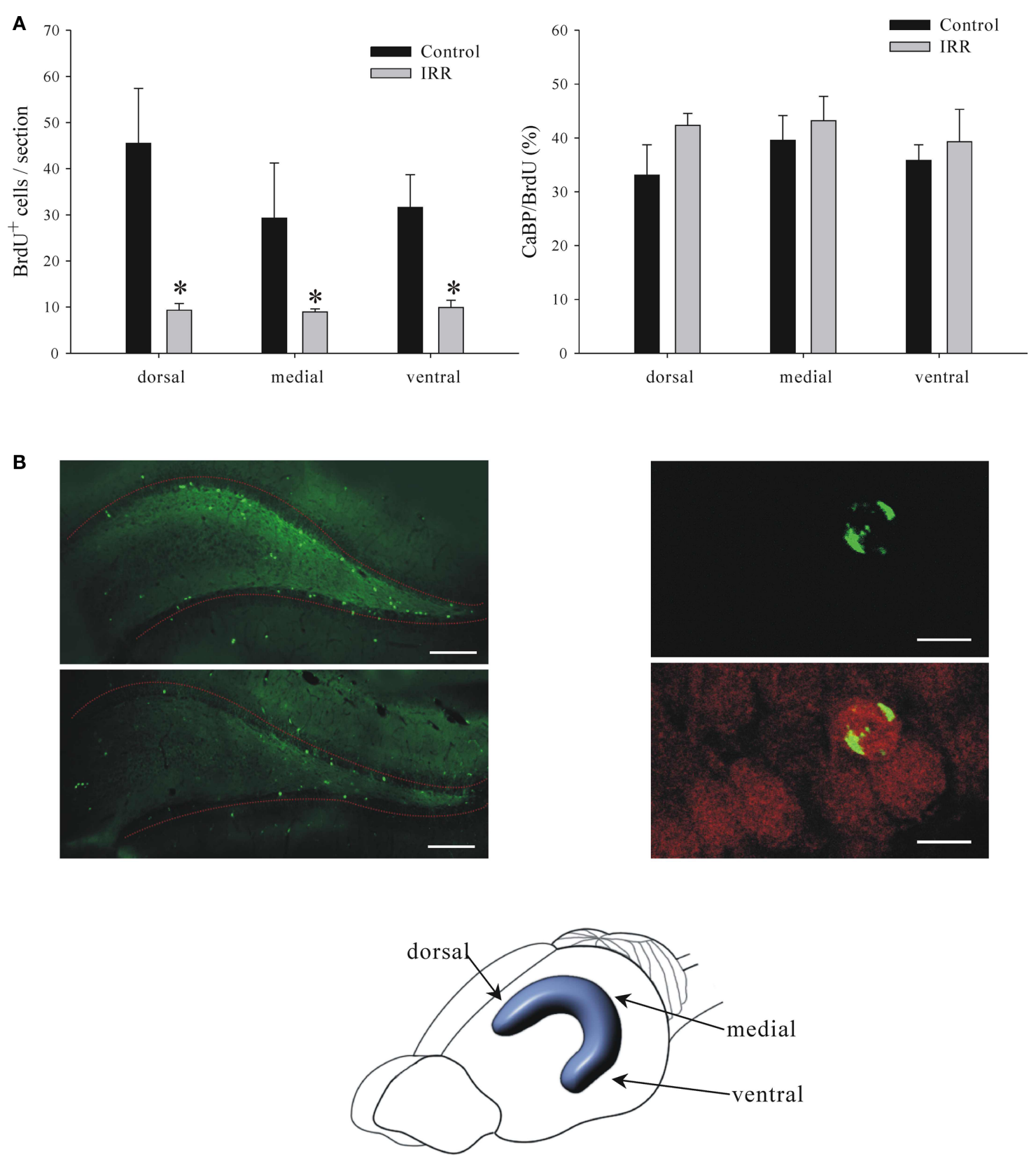

FIGURE 6 | Details of reduced neurogenesis at 6 weeks after irradiation (IRR). (A) Left. Graph shows numbers of BrdU+ cells in dorsal, medial, and ventral portions of the dentate gyrus. Reduction of neurogenesis in irradiated animals was equal and significant in all three regions (two-way ANOVA, $P=0.001$ ). Right. Graph shows ratios of $\mathrm{CaBP}+$ cells to the total number of $\mathrm{BrdU}+$ cells in the three regions. There were no significant differences between regions or treatment (two-way ANOVA, $P=0.645$ ) indicating that the irradiation is equally effective in all three regions and there is no effect on cell maturation. (B) Panels show examples of images stained for BrdU (left panel) or BrdU and CaBP (right panel). BrdU is shown in green and $\mathrm{CaBP}$ is shown in red. Calibration bars are 200 and $15 \mu \mathrm{m}$ in left and right panels, respectively. the initial damage. This partial recovery seems to take the course of de novo renewal of the young cell population. Accordingly, the DCX+ cells observed at 1 week of recovery are very similar to 1 -week-old neurons in their normal development (McDonald and Wojtowicz, 2005). Such cells are characterized by clustering and posses short, stubby dendrites extending horizontally, in parallel to the hilar/GCL border (McDonald and Wojtowicz, 2005; Seki et al., 2007). More developed neurons with long dendrites are missing. In contrast, at 6 weeks the cells appear to include a normal range of shapes and sizes typical for the young neuronal population. At higher doses there is no rebound and ultimately the damage is permanent.

In summary, our results demonstrate the usefulness of the irradiation approach to studies of adult neurogenesis. With the targeted application of the radiation, the full dose was centered 
in the $5 \mathrm{~mm} \times 5 \mathrm{~mm} \times 5 \mathrm{~mm}$ voxel of the brain tissue that comprised both hippocampi. There was an inevitable "spill-over" effect that included the surrounding brain tissue but the $80-60 \%$ isodose levels declined sharply within the approximate volume of $10 \mathrm{~mm} \times 10 \mathrm{~mm} \times 10 \mathrm{~mm}$. The effects were dose-dependent and easily verifiable with post hoc immunohistochemistry. The effects of irradiation were uniform along the dorso-ventral axis of the hippocampus with equal effect on the number of BrdU+ cells in the dorsal, medial, and ventral regions. This result serves to verify the accuracy of our irradiation targeting procedure. The equal proportions of the $\mathrm{CaBP} / \mathrm{BrdU}$-labeled cells further show that the cells spared by irradiation develop normally according the same time line as that observed in control animals (Figure 6).

These results may be used in assessing possible collateral damage caused by radiotherapy and other types of treatment or imaging procedures involving radiation. It would appear that doses as small as 100 cGy (0.1 Gy) could cause noticeable damage in the

\section{REFERENCES}

Barani, I. J., Benedict, S. H., and Lin, P.-S. (2007). Neural stem cells: implications for the conventional radiotherapy of central nervous system malignancies. Int. J. Radiat. Oncol. Biol. Phys. 68, 324-333.

Ben Abdallah, N. M.-B., Slomianka, L., and Lipp, H.-P. (2007). A reversible effect of X-irradiation on proliferation, neurogenesis and cell death in the dentate gyrus of adult mice. Hippocampus 17, 1230-1240.

Bernad, D., McAndrews, M., Kong, I., Becker, S., Shah, M., Wojtowicz, M., Cusimano, M., Laperriere, N., Mikulis, D., and Menard, C. (2010). The effects of low dose Hippocampal radiation exposure on memory in patients receiving stereotactic radiosurgery for benign neurological disorders. American Society for Radiation Oncology (ASTRO) Annual Meeting. Int. J. Radiat. Oncol. Biol. Phys. 78(Suppl. 3), 1066; S169.

Canales, J. J. (2007). Adult neurogenesis and the memories of drug addiction. Eur. Arch. Psychiatry Clin. Neurosci. 257, 261-270.

Cohen, N. J., and Eichenbaum, H. (1993). Memory, Amnesia and the Hippocampal System. Cambridge: The MIT Press.

Dash, P. K., Mach, S. A., and Moore, A. N. (2001). Enhanced neurogenesis in the rodent hippocampus following traumatic brain injury. J. Neurosci. Res. 63, 313-319.

Eisch, A. J., Barrot, M., Schad, C. A., Self, D. W., and Nestler, E. J. (2000). Opiates inhibit neurogenesis in the adult rat hippocampus. Proc. Natl. Acad. Sci. U.S.A. 97, 7579-7584.
Encinas, J. M., Vazquez, M. E., Switzer, R. C., Chamberland, D. W., Nick, H., Levine, H. G., Scarpa, P. J., Enikolopov, G., and Steindler, D. A. (2008). Quiescent adult neural stem cells are exceptionally sensitive to cosmic radiation. Exp. Neurol. 210, 274-279.

Hernandez-Rabaza, V., Llorens-Martin, M.V., Ferragud,A., Velazquez-Sanches, C., Arcusa, A., Gomez-Pinedo, U., Perez-Villalba, A., Rosello, J., Trejo, J. L., Barcia, J.A., and Canales, J. J. (2009). Inhibition of adult hippocampal neurogenesis disrupts contextual learning but spares spatial working memory, long-term conditional rule retention and spatial reversal. Neuroscience 159, 59-68.

Kee, N., Preston, E., and Wojtowicz, J. M. (2001). Enhanced neurogenesis after transient ischemia in the dentate gyrus of the rat. Exp. Brain Res. 136, 313-320.

Kee, N., Sivalingam, S., Boonstra, R., and Wojtowicz, J. M. (2002). The utility of Ki-67 and BrdU as proliferative markers of adult neurogenesis. J. Neurosci. Methods 115, 97-105.

Koh, E.-S., Laperriere, N., Bernstein, M., Jaffray, D., and Menard, C. (2006). Cranial stereotactic surgery: overview of current technology and clinical practice. Oncol. Rounds 6, 1-5.

Laack, N. N., and Brown, P. D. (2004). Cognitive sequelae of brain radiation in adults. Semin. Oncol. 31, 702-713.

McDonald, H. Y., and Wojtowicz, J. M. (2005). Dynamics of neurogenesis in the dentate gyrus of adult rats. Neurosci. Lett. 385, 70-75.

Mizumatsu, S., Monje, M. L., Morhardt, D. R., Rola, R., Palmer, T. D., and Fike, J. R. (2003). Extreme sensitivity of adult neurogenesis to low doses

neurogenic regions of the hippocampus. This in turn could cause deficits in learning and memory as well as possible changes in sensitivity of the subjects to antidepressants. Some aspects of depression, schizophrenia, and opioid-dependence have been linked to adult neurogenesis (Eisch et al., 2000; Santarelli et al., 2003; Reif et al., 2006; Canales, 2007). Reduced neurogenesis is expected to negatively affect the symptoms and treatment of these conditions. A proof of principle evidence has recently emerged from a study Dr. C. Menard and colleagues who reported memory deficits in patients undergoing radiation therapy at doses as low as $0.75 \mathrm{~Gy}$ (Bernard et al., 2010).

\section{ACKNOWLEDGMENTS}

We thank M. Khan, Y. Wang and J. Zhang for technical assistance in the study. Ms. Lulu Gao helped with immunohistochemical staining for microglia. This research was funded by operating grants from CIHR and NSERC.

of X-irradiation. Cancer Res. 63, 4021-4027.

Monje, M. L., Mizumatsu, S., Fike, J. R. and Palmer, T. D. (2002). Irradiation induces neural precursor-cell dysfunction. Nat. Med. 8, 955-962.

Monje, M. L., and Palmer, T. D. (2003) Radiation injury and neurogenesis. Curr. Opin. Neurol. 16, 129-134.

Monje, M. L., Toda, H., and Palmer, T. D. (2003). Inflammatory blockade restores adult hippocampal neurogenesis. Science 302, 1760-1765.

Noonan, M.A., Bulin, S.E., Fuller,D.C., and Eisch, A. J. (2010). Reduction of adult hippocampal neurogenesis confers vulnerability in an animal model of cocaine addiction. J. Neurosci. 30, 304-315.

Peissner, W., Kocher, M., Treurer, H., and Gillardon, F. (1999). Ionizing radiation-induced apoptosis of proliferating stem cells in the dentate gyrus of the adult rat hippocampus. Mol. Brain Res. 71, 61-68.

Reif, A., Fritzen, S., Finger, M., Strobel, A. Lauer, M., Schmitt, A., and Lesch, K. P. (2006). Neural stem cell proliferation is decreased in schizophrenia, but not in depression. Mol. Psychiatry 11, 514-522.

Rola, R., Raber, J., Rizk, A., Otsuka, S. VandenBerg, S. R., Morhardt, D. R., and Fike, J. R. (2004). Radiationinduced impairment of hippocampal neurogenesis is associated with cognitive deficits in young mice. Exp. Neurol. 188, 316-330.

Santarelli, L., Saxe, M., Gross, C., Surget, A., Battaglia, F., Dulawa, S., Weisstaub, N., Lee, J., Duman, R. S., Arancio, O., Belzung, C., and Hen, R. (2003). Requirement of hippocampal neurogenesis for the behavioral effects of antidepressants. Science 301, 805-809.
Saxe, M. D., Battaglia, F., Wang, J.-W., Melleret, G., David, D. J., Monckton, J. E., Garcia, A. D. R., Sofroniev, M. V., Kandel, E. R., Santarelli, L., Hen, R., and Drew, M. R. (2006). Ablation of hippocampal neurogenesis impairs contextual fear conditioning and synaptic plasticity in the dentate gyrus. Proc. Natl. Acad. Sci. U.S.A. 103, 17501-17506.

Seki, T., and Arai, Y. (1993). Highly polysialylated neural cell adhesion molecule (NCAM-H) is expressed by newly generated granule cells in the dentate gyrus of the adult rat. J. Neurosci. 13, 2351-2358.

Seki, T., Namba, T., Mochizuki, H., and Onodera, M. (2007). Clustering, migration, and neurite formation of neural precursor cells in the adult rat hippocampus. J. Comp. Neurol. 502, 275-290.

Sharp, F. R., Liu, J., and Bernabeu, R. (2002). Neurogenesis following brain ischemia. Dev. Brain Res. 134, 23-30.

Snyder, J.S., Radik, R., Wojtowicz, J.M., and Cameron, H. A. (2008). Anatomical gradients of neurogenesis and activity: young neurons in the ventral dentate gyrus are activated by water maze training. Hippocampus 19, 360-370.

Tada, E., Parent, J. M., Lowenstein, D. H. and Fike, J. R. (2000). X-irradiation causes a prolonged reduction in cell proliferation in the dentate gyrus of adult rats. Neuroscience 99, 33-41.

Tan, Y.-F., Preston, E., and Wojtowicz, J. M. (2010). Enhanced postischemic neurogenesis in aging rats. Front. Neurosci. 4:163. doi: 10.3389/ fnins.2010.00163

Winocur, G., Wojtowicz, J. M., Sekeres, M., Snyder, J. S., and Wang, S. (2006) 
Inhibition of neurogenesis interferes with hippocampal-dependent memory function. Hippocampus 16 , 296-304.

Wojtowicz, J. M. (2006). Irradiation as an experimental tool in studies of adult neurogenesis. Hippocampus 16, 261-266.

Wojtowicz, J. M., Askew, M. L., and Winocur, G. (2008). The effects of running and inhibiting adult neurogenesis on learning and memory in rats. Eur. J. Neurosci. 27, 1494-1502.

Wojtowicz, J.M., and Kee, N. (2006). BrdU assay for neurogenesis in rodents. Nat. Protocols 1, 1399-1405.

Conflict of Interest Statement: The authors declare that the research was conducted in the absence of any commercial or financial relationships that could be construed as a potential conflict of interest.

Received: 24 January 2011; accepted: 07 April 2011; published online: 21 April 2011.

Citation: Tan Y.-F, Rosenzweig S, Jaffray $D$ and Wojtowicz JM (2011) Depletion of new neurons by image guided irradiation. Front. Neurosci. 5:59. doi: 10.3389/ fnins.2011.00059
This article was submitted to Frontiers in Neurogenesis, a specialty of Frontiers in Neuroscience.

Copyright (C) 2011 Tan, Rosenzweig, Jaffray and Wojtowicz. This is an open-access article subject to a non-exclusivelicense between the authors and Frontiers Media SA, which permits use, distribution and reproduction in other forums, provided the original authors and source are credited and other Frontiers conditions are complied with. 